\title{
Glutamate residues in the putative transmembrane region are required for the function of the VirS sensor histidine kinase from Clostridium perfringens
}

\author{
Jackie K. Cheung and Julian I. Rood
}

Bacterial Pathogenesis Research Group, Department of Microbiology, Monash University, 3800 Clayton, Australia
Author for correspondence: Julian I. Rood. Tel: +61 39905 4825. Fax: +6139905 4811. e-mail: Julian.Rood@med.monash.edu.au

\begin{abstract}
The causative agent of gas gangrene, Clostridium perfringens, is a Grampositive anaerobe which produces a number of extracellular toxins and enzymes. The production of several of these toxins is regulated by the VirS/VirR two-component signal transduction system. The sensor histidine kinase, VirS, contains motifs that are conserved amongst sensor histidine kinases, although not in the same relative positions. In this study, the conserved histidine residue (H255), the GXGL and DXGXG motifs, and two glutamate residues located in putative transmembrane domains were altered by site-directed mutagenesis to examine their significance for VirS function. Introduction of the mutated virS genes into the virS: :Tn916 mutant, JIR4000, showed that the altered virs genes were not able to complement the host mutation. These results demonstrate that the conserved motifs, including the cytoplasmic DXGXG motif which is located between the putative transmembrane domains 4 and 5 , are functional. Furthermore, it is concluded that charged residues located within two of these transmembrane domains are also required for the structural or functional integrity of the VirS sensor kinase.
\end{abstract}

Keywords: Clostridium perfringens, two-component, signal transduction, sensor histidine kinase, transmembrane domain

\section{INTRODUCTION}

Bacteria live in an ever-changing environment, and to survive they must constantly monitor their surroundings and respond quickly and efficiently to various environmental cues. The most common adaptive response involves two-component signal transduction systems. These regulatory networks have been identified in both Gram-positive and Gram-negative bacteria and have been found to control a variety of environmental responses, including chemotaxis, nitrogen regulation, phosphate regulation, osmoregulation, antibiotic resistance and virulence (Stock et al., 1989, 1995). Twocomponent signal transduction systems usually consist of two proteins, a membrane-associated sensor histidine kinase and its cognate cytoplasmic response regulator.

In general, sensor histidine kinases can be divided into two domains. The more conserved domain extends over

Abbreviation: RT, reverse transcriptase. approximately 250 amino acids and is usually located at the cytoplasmic C-terminus of the protein (Albright et al., 1989). Sequence alignments of sensor kinases reveal that there are five regions that show stronger conservation. These motifs include an invariant histidine residue that acts as the site of autophosphorylation and GXGL, DXGXG, $\mathrm{N}$ and $\mathrm{F}$ motifs, which have been proposed to form a nucleotide-binding surface (Parkinson \& Kofoid, 1992; Stock et al., 1995). The $\mathrm{N}$-terminal domain is less well conserved than the C-terminus and most sensor kinases contain transmembrane regions which have been postulated to function as membrane receptors (Stock et al., 1989). In general, these proteins contain two N-terminal membrane-spanning segments (Parkinson \& Kofoid, 1992), with the extracytoplasmic loop between the transmembrane segments proposed to be the site of interaction with the environmental stimulus (Stock et al., 1995).

In the Gram-positive anaerobe Clostridium perfringens, 


\section{EXTERIOR}

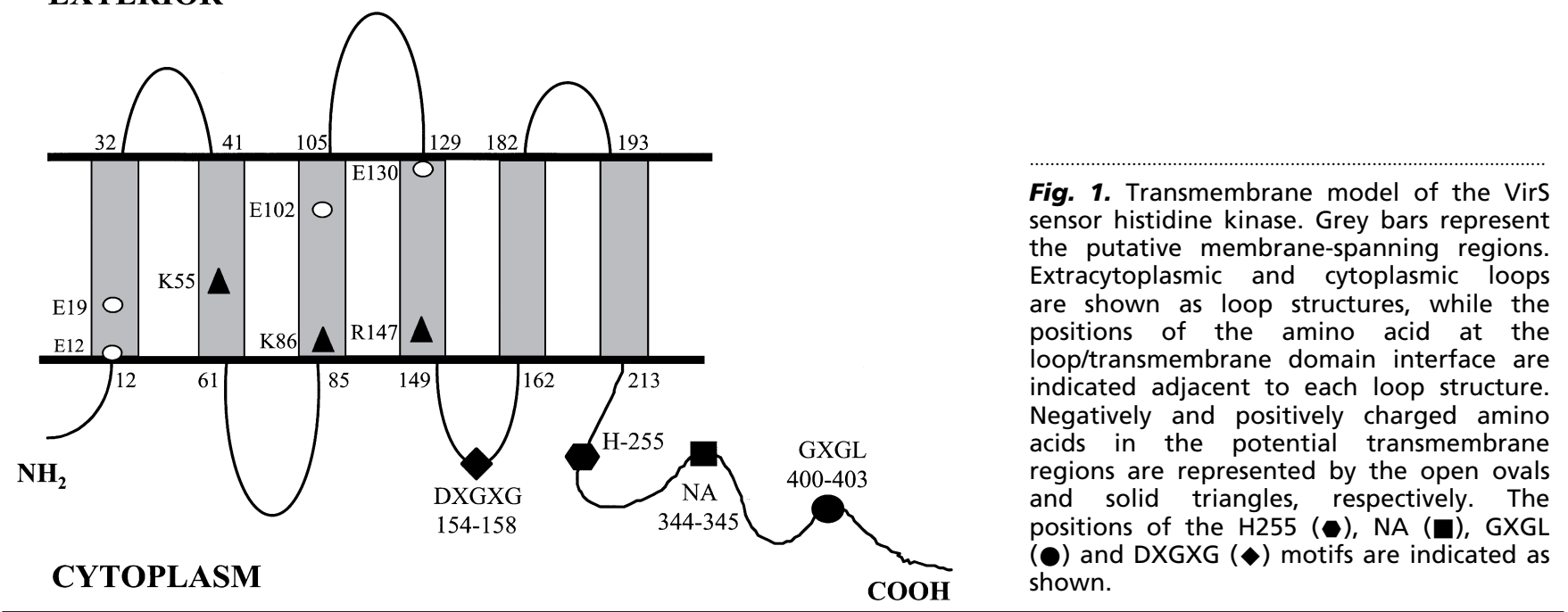

which is the causative agent of gas gangrene (Rood \& Cole, 1991), toxin production is regulated by the VirS/VirR two-component signal transduction system (Lyristis et al., 1994; Shimizu et al., 1994). The virR and virS genes form an operon (Ba-Thein et al., 1996), with virR encoding the cytoplasmic response regulator and virS encoding the sensor histidine kinase (Lyristis et al., 1994). Both proteins contain motifs and residues that are conserved amongst their two-component homologues. However, the VirS sensor histidine kinase differs in two ways. First, it contains six putative transmembrane regions, whereas most sensor kinases have two putative membrane-spanning regions. Secondly, the DXGXG motif is postulated (Lyristis et al., 1994) to be in a cytoplasmic loop between the fourth and fifth putative transmembrane segments (Fig. 1); this motif is generally situated near the GXGL motif in the cytoplasmic Cterminal catalytic domain in most sensor kinases (Parkinson \& Kofoid, 1992).

The VirS/VirR system differentially regulates the production of several of the toxins potentially involved in pathogenesis and virulence, namely $\alpha$-toxin (phospholipase $\mathrm{C})$, perfringolysin $\mathrm{O}$ ( $\theta$-toxin), collagenase ( $\kappa$-toxin), sialidase and protease (Lyristis et al., 1994; Shimizu et al., 1994). It is proposed that upon the detection of an as yet unknown environmental or growth phase stimulus by the $\mathrm{N}$-terminal transmembrane regions, the VirS protein undergoes a conformational change. This change initiates the autophosphorylation process, whereby a phosphoryl group is transferred from ATP to the conserved $\mathrm{H} 255$ residue. The phosphorylated VirS protein then acts as a phosphodonor, leading to the phosphorylation of the D57 residue of VirR. The resultant activated VirR molecule subsequently regulates the transcription of the various target genes, leading to increased toxin production (Lyristis et al., 1994; Rood \& Lyristis, 1995).

In this study, the conserved histidine residue $\mathrm{H} 255$, the two glycine-rich motifs GXGL and DXGXG, and two glutamate residues in the putative transmembrane region were changed by site-directed mutagenesis. To assess the effect of these mutations and therefore the functional significance of the original amino acids, complementation assays were carried out by introducing the mutated genes into the virS: : Tn916 mutant JIR4000 (Lyristis et al., 1994).

\section{METHODS}

Bacterial strains, plasmids and growth media. Bacterial strains and plasmids used in this study are listed in Table 1. Escherichia coli strains were cultured at $37^{\circ} \mathrm{C}$ on/in $2 \times \mathrm{YT}$ agar or broth or in SOC broth (Sambrook et al., 1989), supplemented with $100 \mu \mathrm{g}$ ampicillin $\mathrm{ml}^{-1}$ or $150 \mu \mathrm{g}$ erythromycin $\mathrm{ml}^{-1}$. C. perfringens strains were grown at $37^{\circ} \mathrm{C}$ in trypticase-peptone-glucose (TPG) broth (Rood et al., 1978), Brain Heart Infusion (BHI) broth (Oxoid), fluid thioglycolate medium (FTG) (Difco) or nutrient agar (Rood, 1983) supplemented with $50 \mu \mathrm{g}$ erythromycin $\mathrm{ml}^{-1}$ or $10 \mu \mathrm{g}$ tetracycline $\mathrm{ml}^{-1}$. When screening for perfringolysin $\mathrm{O}$ production, C. perfringens transformants were grown on horse blood agar (HBA) (Lyristis et al., 1994). All agar cultures of C. perfringens were incubated in an atmosphere of $10 \%(\mathrm{v} / \mathrm{v}) \mathrm{H}_{2}, 10 \%(\mathrm{v} / \mathrm{v})$ $\mathrm{CO}_{2}$ in $\mathrm{N}_{2}$.

Molecular techniques. Plasmid DNA from E. coli cells was routinely isolated by an alkaline lysis method (Morelle, 1989). When used for sequencing, DNA was obtained using the modified mini alkaline-lysis/PEG precipitation procedure outlined in the PRISM Ready Reaction DyeDeoxy Terminator Cycle Sequencing kit (Applied Biosystems). C. perfringens plasmid DNA was isolated using a modified Magic Mini Prep kit method (Promega) (Lyristis et al., 1994). Rubidiumchloride-competent E. coli cells were prepared and transformed as described previously (Hanahan, 1985). Transformation of electrocompetent E. coli cells (Smith et al., 1990) was carried out with a Bio-Rad Gene Pulser in $0 \cdot 1 \mathrm{ml}$ cuvettes (Bio$\mathrm{Rad}$ ) under conditions outlined by the manufacturer. Electrocompetent C. perfringens cells were prepared and transformed as before (Scott \& Rood, 1989).

Nucleotide sequence analysis. Nucleotide sequences were determined by use of a PRISM Big Dye Terminator Cycle 
Table 1. Relevant strains and plasmids

\begin{tabular}{|c|c|c|}
\hline Strain/plasmid & Characteristics & Reference/origin \\
\hline \multicolumn{3}{|l|}{ Strains } \\
\hline \multicolumn{3}{|l|}{ E. coli } \\
\hline $\mathrm{DH} 5 \alpha$ & $\begin{array}{l}\mathrm{F}^{-} 80 \mathrm{~d} l a c Z \Delta \mathrm{M} 15 \Delta(\operatorname{lac} Z Y A-\arg F) \mathrm{U} 169 \text { endA1 recA1 hsdR17 }\left(\mathrm{r}_{\mathrm{k}}^{-} \mathrm{m}_{\mathrm{k}}^{+}\right) \\
\text {deoR thi-1 supE44 gyrA96 relA1 }\end{array}$ & Bethesda Research Laboratories \\
\hline \multicolumn{3}{|l|}{ C. perfringens } \\
\hline JIR325 & Strain $13 \mathrm{Nal}^{\mathrm{R}} \mathrm{Rif}^{\mathrm{R}}$ & Lyristis et al. (1994) \\
\hline JIR4025 & JIR325 Rifs & Lyristis et al. (1994) \\
\hline JIR4000 & JIR4025 virS: : Tn916 & Lyristis et al. (1994) \\
\hline \multicolumn{3}{|l|}{ Plasmids } \\
\hline pBluescript II SK(+) & Cloning vector, $\mathrm{Ap}^{\mathrm{R}}$ & Stratagene \\
\hline pJIR751 & C. perfringens-E. coli shuttle vector, $\mathrm{Em}^{\mathrm{R}}$ & Bannam \& Rood (1993) \\
\hline pJIR888 & 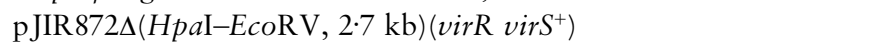 & Lyristis et al. (1994) \\
\hline pJIR1364 & pBluescript II SK $(+) \Omega($ SalI/PstI:pJIR $888,1 \cdot 6 \mathrm{~kb})$ & Recombinant \\
\hline pJIR1216 & 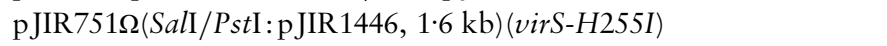 & Lyristis $(1996)$ \\
\hline pJIR1481 & 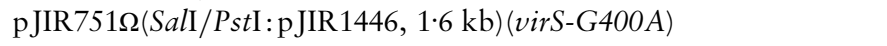 & Cloned site-directed mutant \\
\hline pJIR1483 & 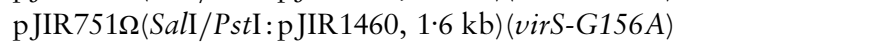 & Cloned site-directed mutant \\
\hline pJIR1506 & 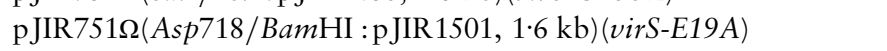 & Cloned site-directed mutant \\
\hline pJIR1712 & 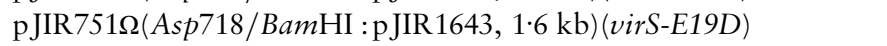 & Cloned site-directed mutant \\
\hline pJIR1715 & 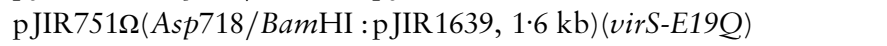 & Cloned site-directed mutant \\
\hline pJIR1507 & 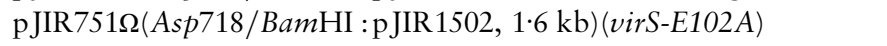 & Cloned site-directed mutant \\
\hline pJIR1713 & 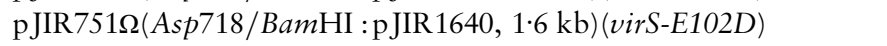 & Cloned site-directed mutant \\
\hline pJIR1714 & 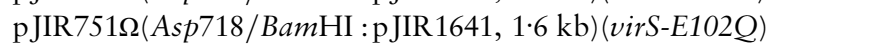 & Cloned site-directed mutant \\
\hline
\end{tabular}

Sequencing Ready Reaction kit with AmpliTaq DNA Polymerase, FS (Applied Biosystems) according to the manufacturer's instructions. Sequencing samples were resolved and analysed on a 373 DNA STRETCH Sequencer (Applied Biosystems). Sequence analysis was performed using the Sequencher 3.0 software (GeneCodes). Transmembrane protein structure was predicted by use of TopPred II software (Claros \& von Heijne, 1994).

Construction of recombinant plasmids. Plasmid DNA was digested in the presence of the appropriate restriction enzyme(s) and digestion buffer (Boehringer Mannheim; New England Biolabs) under the conditions specified by the manufacturers. Insert and vector DNA were isolated using the BRESA-CLEAN Nucleic Acid Purification kit (Bresatec). Vector and insert DNA were ligated with T4 DNA ligase (3 units $\mu \mathrm{l}^{-1}$; Promega) at $16^{\circ} \mathrm{C}$ overnight. The target plasmid for site-directed mutagenesis, pJIR1364, was obtained by cloning the $1.6 \mathrm{~kb}$ SalI-PstI fragment of pJIR888, which contained the virS gene, into pBluescript II $\mathrm{SK}(+)$ (Stratagene).

Site-directed mutagenesis. Site-directed mutagenesis of the virS gene was carried out by the unique site replacement method (Deng \& Nickoloff, 1992) with a U. S. E. Mutagenesis kit (Pharmacia Biotech) by a modification of the manufacturer's instructions. After annealing the selection and mutagenic primers to the plasmid template, synthesis of a mutant DNA strand was carried out at $42{ }^{\circ} \mathrm{C}$ for $1 \mathrm{~h}$. The second round of restriction enzyme selection was performed in a total volume of $20 \mu \mathrm{l}$. The digested DNA was then used to transform rubidium-chloride-treated competent $E$. coli cells; the cells were heat-shocked at $37^{\circ} \mathrm{C}$ for $2 \mathrm{~min}$ before inoculation into SOC broth. Plasmids were screened using restriction analysis and the desired mutations were verified by nucleotide sequence analysis. The oligonucleotide primers used for mutagenesis (Table 2) were synthesized on an Applied Biosystems 394 DNA/RNA Synthesizer. All mutated virS genes were completely sequenced to confirm that no additional mutations had been introduced.

Perfringolysin $\mathbf{O}$ assays. Perfringolysin $\mathrm{O}$ activity was determined by measuring the haemolysis of horse erythrocytes. C. perfringens cultures and supernatants were obtained as described previously (Awad et al., 1995). Haemolysin assays were performed by a doubling-dilution assay as before (Stevens et al., 1987), except that $5 \mathrm{mM}$ DTT was used. The titre was defined as the reciprocal of the last well that showed complete haemolysis.

Isolation of RNA. To obtain cells for RNA extraction, $3.0 \mathrm{ml}$ of each overnight FTG culture of the relevant $C$. perfringens strain was subcultured into $20 \mathrm{ml} \mathrm{BHI}$ broth and grown at $37{ }^{\circ} \mathrm{C}$ until late exponential phase. Cells were harvested by centrifugation at $16300 \mathrm{~g}$ and the supernatant was discarded. The cells were resuspended and incubated in $1 \mathrm{ml}$ lysis solution (10 mM Tris/ $\mathrm{HCl}, \mathrm{pH} 7.5 ; 0.4 \mathrm{M}$ sucrose; $5 \mathrm{mg}$ lysozyme $\mathrm{ml}^{-1}$ ) for $15 \mathrm{~min}$ at $37^{\circ} \mathrm{C}$, harvested by centrifugation, and the supernatant was removed. Total RNA was then isolated using TRIzol Reagent (Gibco-BRL/Life Technologies) as described previously (La Fontaine \& Rood, 1996), with a few modifications. To remove contaminating DNA, RNA samples were treated with 1 unit RQ1 RNase-free DNase I (Promega) in the presence of $0 \cdot 1$ vol. $10 \times$ DNase I buffer $(40 \mathrm{mM}$ Tris/HCl, pH 7.9; $10 \mathrm{mM} \mathrm{NaCl} ; 6 \mathrm{mM}$ $\mathrm{MgCl}_{2} ; 10 \mathrm{mM} \mathrm{CaCl}_{2}$ ) and 40 units RNasin RNase Inhibitor (Promega) for $1 \mathrm{~h}$ at $37^{\circ} \mathrm{C}$. Samples were then re-extracted with TRIzoL according to the manufacturer's instructions, but using only half the original reagent volumes.

RT-PCR. Reverse transcription was carried out using $2 \mu \mathrm{g}$ RNA as the template, following the manufacturer's instructions 
Table 2. Oligonucleotide primers used in this study

\begin{tabular}{|lll|}
\hline Primer no. & \multicolumn{1}{c|}{ Sequence $\left(\mathbf{5}^{\prime} \mathbf{- 3}^{\prime}\right)$} & \multicolumn{1}{c|}{ Purpose } \\
\hline 2281 & GGCGGCCGCTCCAGAACTAGTG & Xbal selection primer \\
3537 & CTTATATTTTTAAGGCCAATAGCATGTAAAAAC & G400A mutation \\
3908 & TAAAGTCTCCCTTTGCAATATCTACACG & G156A mutation \\
4622 & CTTACCCATGCTATAATC & E19A mutation \\
5323 & CTTACCCAATCTATAATC & E19D mutation \\
5322 & CTTACCCATTGTATAATC & E19Q mutation \\
4623 & GAAATAGATAGAGCTGCTACTG & E102A mutation \\
5324 & GATAGAGCGTCTACTGTAAGC & E102D mutation \\
5325 & GAAATAGATAGAGCTTGTACTG & E102Q mutation \\
1001 & GATCTCATTAATATTTTGGT & RT-PCR \\
1495 & TACATATATCAAAGTACTCAAT & RT-PCR \\
\hline
\end{tabular}

(Promega), with minor modifications. The reaction was performed at $42^{\circ} \mathrm{C}$ for $50 \mathrm{~min}$, followed by heat inactivation of the AMV reverse transcriptase at $70^{\circ} \mathrm{C}$ for $15 \mathrm{~min}$.

RT and PCR reactions were carried out consecutively. PCR was performed in a total volume of $25 \mu \mathrm{l}$; the reaction mixture consisted of $0.2 \mathrm{vol}$. of the RT reaction, $0.5 \mu \mathrm{M}$ of each internal virS primer (Table 2), 0.1 vol. $10 \times$ PCR buffer (Boehringer Mannheim), 2.5 units Taq DNA polymerase (Boehringer Mannheim) and sterile distilled water. Reactions were carried out in a GeneAmp PCR System 2400 (Perkin Elmer), where the $94^{\circ} \mathrm{C}$ denaturation $(1 \mathrm{~min}), 50{ }^{\circ} \mathrm{C}$ annealing $(2 \mathrm{~min})$ and $72{ }^{\circ} \mathrm{C}$ extension $(3 \mathrm{~min})$ steps were carried out for 30 cycles. The final cycle consisted of 2 min annealing and 5 min extension at the temperatures indicated. RT-PCR products were resolved and visualized by agarose gel electrophoresis.

\section{RESULTS}

\section{Functional analysis of the conserved $\mathrm{H} 255$ residue and GXGL motif}

To confirm that the VirS protein was a functional sensor histidine kinase we used site-directed mutagenesis to examine the functional significance of the conserved H255 residue and GXGL motif. The proposed site of autophosphorylation, $\mathrm{H} 255$, was changed to isoleucine to observe the effect of a change from a basic hydrophilic amino acid to a hydrophobic amino acid that could not act as a phosphoacceptor. The VirS GXGL motif has been postulated to be the conserved nucleotide binding site (Lyristis et al., 1994). To determine whether this motif was functional in VirS, the first glycine residue of the putative GXGL motif, G400, was changed to alanine. The target plasmid, pJIR1364, was subjected to mutagenesis as described in Methods and potential H255I and G400A mutants were identified by restriction endonuclease digestion and preliminary DNA sequencing. The virS gene regions of potential mutants were then completely sequenced and no additional mutations were found.
To examine the effect of the H255I and G400A mutations on VirS function in the native C. perfringens host, the mutated virS genes were subcloned into an $E$. coli-C. perfringens shuttle vector, pJIR751 (Bannam \& Rood, 1993). The resultant constructs, pJIR1216 and pJIR1481, respectively, were then introduced into the C. perfringens strain, JIR 4000. This strain carries a chromosomal virS mutation which alters its toxin production profile to the extent that $\alpha$-toxin and collagenase production are reduced and perfringolysin $\mathrm{O}$ production is completely eliminated (Lyristis et al., 1994). Complementation of the mutation with a plasmid carrying a wild-type virS gene results in restoration of the ability to produce perfringolysin $\mathrm{O}$, depending upon the level of expression of the wild-type gene. This complementation can be readily observed as a zone of haemolysis on HBA.

Unlike the $v i r S^{+}$positive control, pJIR888, the mutated virS plasmids, pJIR1216 and pJIR1481, did not complement the chromosomal virS mutation. No zone of haemolysis was observed on HBA. This observation was confirmed by carrying out quantitative perfringolysin $\mathrm{O}$ assays using culture supernatants (Table 3 ). As expected, the wild-type strain JIR325 produced high levels of perfringolysin $\mathrm{O}$, while the mutant strain JIR4000 and the mutant strain carrying the shuttle plasmid, JIR4000(pJIR751), did not produce detectable levels of haemolysis. Note that lower levels of perfringolysin $\mathrm{O}$ activity were observed in supernatants from JIR4000(pJIR888), presumably because this plasmid does not have the native virRS promoter. These results were in agreement with the phenotypes observed on HBA.

Although these results indicated that the H255I and G400A mutations had altered the function of VirS, it was necessary to confirm that the mutated gene was still transcribed. Total RNA was isolated from $C$. perfringens strains carrying either the control plasmid or the mutated virS genes. Following DNase I treatment, the RNA samples were used as templates in RT-PCR experiments. A positive DNA control, which used the 
Table 3. Effect of site-directed mutagenesis on perfringolysin O production

\begin{tabular}{|c|c|c|c|}
\hline Strain & Plasmid VirS mutation & Nucleotide change & PfoA titre* \\
\hline JIR325 & Wild-type & & $8 \cdot 2 \pm 0 \cdot 4$ \\
\hline JIR4000 & Chromosomal virS mutant & & $<\overline{1} \cdot 0$ \\
\hline JIR4000(pJIR751) & Negative control & & $<1 \cdot 0$ \\
\hline JIR4000(pJIR888) & Positive control & & $2 \cdot 8 \pm 0 \cdot 3$ \\
\hline JIR4000(pJIR1216) & $\mathrm{H} 255 \mathrm{I}$ & $\mathrm{CAT} \rightarrow \mathrm{ATC}$ & $<1 \cdot 0$ \\
\hline JIR4000(pJIR1481) & G400A & $\mathrm{GGT} \rightarrow \mathrm{GCT}$ & $<1 \cdot 0$ \\
\hline JIR4000(pJIR1483) & G156A & $\mathrm{GGA} \rightarrow \mathrm{GCA}$ & $<1 \cdot 0$ \\
\hline JIR4000(pJIR1506) & E19A & $\mathrm{GAA} \rightarrow \mathrm{GCA}$ & $<1 \cdot 0$ \\
\hline JIR4000(pJIR1712) & E19D & GAA $\rightarrow$ GAT & $<1 \cdot 0$ \\
\hline JIR4000(pJIR1715) & E19Q & GAA $\rightarrow$ CAA & $<1 \cdot 0$ \\
\hline JIR4000(pJIR1507) & E102A & $\mathrm{GAA} \rightarrow \mathrm{GCA}$ & $<1 \cdot 0$ \\
\hline JIR4000(pJIR1713) & E102D & $\mathrm{GAA} \rightarrow \mathrm{GAC}$ & $<1 \cdot 0$ \\
\hline JIR4000(pJIR1714) & E102Q & $\mathrm{GAA} \rightarrow \mathrm{CAA}$ & $<1 \cdot 0$ \\
\hline
\end{tabular}

* PfoA titre refers to the mean perfringolysin O titre obtained from duplicate assays carried out using supernatants from three independent cultures of each strain.

same oligonucleotide primers and pJIR888 as the template, was included at the PCR stage to amplify a product of the same size as that expected from RT-PCR. As anticipated, RNA samples isolated from JIR4000 derivatives carrying pJIR888, pJIR1216 or pJIR1481 all yielded products of the same size (739 bp) as the positive DNA control (Fig. 2). No PCR product was observed from RNA samples isolated from JIR4000 carrying the shuttle vector $\mathrm{pJIR751.} \mathrm{The} \mathrm{absence} \mathrm{of} \mathrm{a} \mathrm{virS} \mathrm{transcript}$ in this background strain has previously been demonstrated and was attributed to the insertion of Tn916 in the chromosomal virS gene (Ba-Thein et al., 1996), the position of which has been mapped previously (Lyristis et al., 1994). The absence of PCR products in the reactions in which $R T$ was omitted confirmed that the RNA samples were DNA-free and that the products observed were the result of the amplification of cDNA molecules derived from the $\mathrm{RT}$ reaction. These products were isolated, sequenced and confirmed to be the internal virS region, thereby demonstrating that the mutated virS genes were expressed and that the observed effects on VirS function could be attributed to the mutations. Based on these results it was concluded that VirS was a functional sensor histidine kinase and that the $\mathrm{H} 255$ and $\mathrm{G} 400$ residues represented conserved components of the respective motifs.

\section{Functional significance of the DXGXG motif}

The cytoplasmic DXGXG motif of sensor histidine kinases is also involved in nucleotide binding (Parkinson \& Kofoid, 1992). However, this motif appears to be located in the cytoplasmic loop between the fourth and fifth putative transmembrane domains of VirS (Fig. 1). Due to its unusual position in comparison to other sensor histidine kinases, the putative DXGXG motif of VirS was mutated to ensure that it was functional. Sitedirected mutagenesis was used to change the first glycine residue (G156) of the motif to alanine. Sequence analysis of the resultant plasmid confirmed that no additional mutations had been introduced. The mutated virS gene was cloned into the shuttle plasmid to construct pJIR1483, which was then introduced into strain JIR4000 and analysed as before.

The results showed that the mutated virS gene was being expressed, as indicated by RT-PCR (Fig. 2a), and that the G156 residue in the DXGXG motif was required for VirS function, since pJIR1483 was not able to complement the virS mutation of JIR 4000, i.e. there was no detectable haemolytic activity on HBA and no demonstrable perfringolysin $\mathrm{O}$ activity in culture supernatants (Table 3 ). These results implied that despite its unusual position, the DXGXG motif had a functional role in VirS activity.

\section{Role of glutamate residues in the putative transmembrane domains}

Topological analysis of the VirS protein was carried out by use of the TopPred II algorithm (Claros \& von Heijne, 1994). The optimal theoretical conformation, i.e. the highest $\mathrm{K}+\mathrm{R}$ difference, was obtained with a window of 21 amino acid residues and predicted the presence of six transmembrane domains (Fig. 1). Examination of each proposed membrane-spanning region revealed the presence of several charged residues (Fig. 1). Of these amino acids, E19 and E102 were the only negatively charged acidic residues predicted to be well within these domains. Other glutamate residues, E12 and E130, were also found in the putative first and fourth transmembrane domains, respectively. However, these amino acids were situated adjacent or very close to putative cytoplasmic or external regions of the protein.

Since the presence of acidic residues located deeply within putative transmembrane domains is thermodynamically undesirable in a stable membrane protein 
(a)

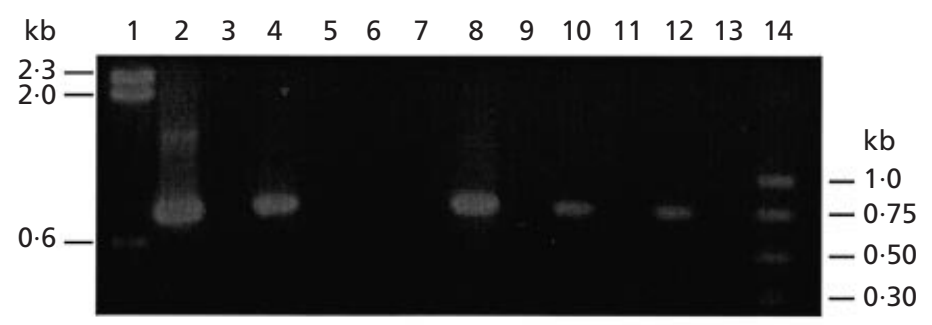

(b)

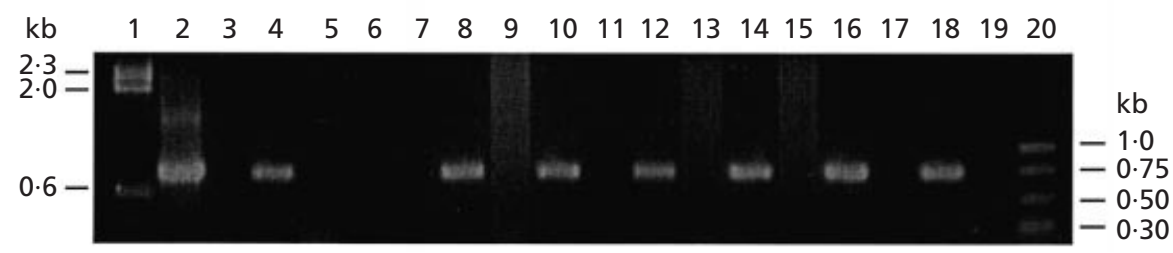

Fig. 2. Agarose gel electrophoresis of RT-PCR products. Lanes: $1, \lambda c / 857$ DNA digested with HindIII; 2 and 3 , the positive [JIR4000(pJIR888)] and negative [JIR4000(pJIR751)] plasmid DNA controls, respectively; 4 and 6, the positive and negative RNA controls, respectively; 5 and 7, the respective no RT controls. The last lane contains PCR markers (Promega). (a) Lanes 8,10 and 12 contain RT-PCR products derived from total RNA isolated from JIR4000 cells harbouring plasmids containing the H255I, G400A and G156A mutations, respectively. Lanes 9, 11 and 13 contain the respective no RT controls. (b) Lanes $8,10,12,14,16$ and 18 contain RT-PCR products derived from total RNA isolated from JIR4000 cells harbouring plasmids carrying the E19A, E19D, E19Q, E102A, E102D and E102Q mutations, respectively. Lanes 9, 11, 13, 15, 17 and 19 contain the respective no RT controls.

our analysis suggested that the E19 and E102 residues may be of functional importance. To examine their significance these residues were each changed to alanine, aspartate and glutamine by site-directed mutagenesis with the oligonucleotides listed in Table 2. The six mutated virS genes (Table 3) were then analysed in the same way as the previous mutations. Again sequence analysis confirmed that no other mutations had been introduced and RT-PCR studies (Fig. 2b) showed that the mutated genes were expressed in C. perfringens. Analysis on HBA and perfringolysin $\mathrm{O}$ assays (Table 3) showed that none of the mutated virS genes complemented the chromosomal virS mutation of JIR 4000 . These results imply that the glutamate residues in the putative transmembrane domains have functional or structural significance in the VirS sensor histidine kinase.

\section{DISCUSSION}

In this study, site-directed mutagenesis of various residues in the kinase and putative transmembrane regions of the sensor histidine kinase, VirS, was carried out to determine if these residues were required for VirS function. Previously, sequence alignment of VirS with other sensor histidine kinases predicted the site of autophosphorylation to be at residue H255 (Lyristis et al., 1994). Studies with well-characterized sensor histidine kinases, such as EnvZ from E. coli (Kanamaru et al., 1990; Yang \& Inouye, 1991, 1993), VirA from Agrobacterium tumefaciens (Jin et al., 1990) and BvgS from Bordetella pertussis (Uhl \& Miller, 1994), have shown that mutation of the conserved histidine residue abolishes the function of the protein. In this study, complementation experiments have shown that a virS gene carrying the $\mathrm{H} 255 \mathrm{I}$ mutation could not complement a chromosomal C. perfringens virS mutation. This result confirmed that $\mathrm{H} 255$ is required for VirS function.

Comparative sequence analysis of VirS also revealed the presence of other conserved regions, namely the N, DXGXG and GXGL motifs (Lyristis et al., 1994). The glycine-rich DXGXG and GXGL motifs have been proposed to be involved in nucleotide binding due to their similarity to the glycine loops that are characteristic of many ATP-binding sites (Parkinson \& Kofoid, 1992). Mutagenesis studies of other sensors have shown that all three motifs are essential for kinase activity (Hsing et al., 1998; Yang \& Inouye, 1993). In this study, virS genes encoding glycine to alanine substitutions in the first glycine-encoding codons of both the DXGXG and GXGL motifs could not complement the virS mutation of the host strain. These results indicate that these glycine residues, and by implication the respective motifs, are of functional or structural importance. By analogy with other sensors it is concluded that the respective VirS motifs are likely to have the same function as the equivalent residues in other histidine kinases.

Recently, the structures of the histidine kinase domains of EnvZ (Tanaka et al., 1998) and the CheA chemotaxis sensor from Thermotoga maritima (Bilwes et al., 1999) were determined. In these kinases, the N, DXGXG and 
GXGL motifs lined the catalytic core that was predicted to be involved in ATP binding (Bilwes et al., 1999; Tanaka et al., 1998). Based on our results it is likely that the DXGXG and GXGL motifs also form part of the catalytic domain of VirS. In almost all sensor histidine kinases, the DXGXG motif is found in close proximity to the GXGL motif and is located in the more conserved C-terminal domain (Parkinson \& Kofoid, 1992; Stock et al., 1989, 1995). However, the VirS DXGXG motif, which we have now shown to be functional, is predicted to be in one of the cytoplasmic loops of the putative transmembrane domains in the variable N-terminal region (Fig. 1). Although not common, the conserved kinase motifs of other sensors have been found in different positions or found to be absent altogether. For example, the histidine residue that acts as the site of autophosphorylation of CheA is located in a region distinct from the conserved kinase domain (Hess et al., 1988), while the $\mathrm{N}$ motif and DXGXG motif are absent in FrzE and AgrORF2, respectively. Similarly, the GXGL motifs are absent in NarX and NarQ (Parkinson \& Kofoid, 1992). We postulate that the conformation of VirS is such that the DXGXG motif, despite its unusual position, can still form the catalytic domain and subsequently bind ATP. Specifically, we propose that the cytoplasmic loop 4-5 must be located in close proximity in the cytoplasm to the conserved C-terminal domain of VirS. Recent studies on EnvZ have indicated that its ATP-binding ability was not affected when the G box (GXGL of VirS) or D/F box (DXGXG of VirS) was altered (Hsing et al., 1998). In contrast, ATP binding was abolished in the N347D mutant of EnvZ (Tanaka et al., 1998). The G box and $\mathrm{D} / \mathrm{F}$ box are presumably important in positioning the phosphate moieties of ATP (Hsing et al., 1998). Therefore, it is possible that mutation of the DXGXG and GXGL motifs of VirS interferes with the positioning of the bound ATP, rather than with actual ATP binding.

The N-terminal domains of sensor histidine kinases are more variable than their C-terminal domains (Parkinson \& Kofoid, 1992). The majority of sensor histidine kinases are membrane-associated and have two transmembrane segments located on either side of a periplasmic domain that presumably interacts with the external stimulus (Parkinson \& Kofoid, 1992; Stock et al., 1989). However, there are several histidine kinases, such as ComP from Bacillus subtilis (Weinrauch et al., 1990), AgrC from Staphylococcus aureus (Lina et al., 1998) and UhpB from E. coli (Island \& Kadner, 1993; Island et al., 1992), which have from six to ten potential transmembrane domains. Similarly, our topological model of VirS predicted the presence of six putative transmembrane domains, with the $\mathrm{N}$ - and $\mathrm{C}$ termini on the cytoplasmic side of the membrane (Fig. 1). Amino acid sequence analysis of the membranespanning segments revealed the presence of two negatively charged glutamate residues that were clearly located within two putative transmembrane domains. These residues were targeted in our mutagenesis experiments because the presence of an acidic amino acid in a hydrophobic membrane region is highly unfavourable for thermodynamic reasons (Lee et al., 1992).

We postulated that the presence of the E19 and E102 residues in putative transmembrane regions implied that they had a role in VirS activity. Evidence in support of this hypothesis was obtained from the mutagenesis experiments. These results showed that when these glutamate residues were changed to alanine, aspartate or glutamine, the mutant VirS proteins were not functional. Glutamate was specifically required at these positions since replacement with an amino acid of similar charge, aspartate, or of similar size, glutamine, did not result in complementation. However, the function of these residues is as yet unknown, and we do not know if these glutamates are of structural or functional significance. It is possible that these mutations interfered with the ability of VirS to undergo a conformational change in the transmembrane domain that is required for signal transfer between the external receptor domain of VirS and the C-terminal kinase domain. Unfortunately, transmembrane signalling in bacteria is poorly understood and what little is known is gleaned from work on the methyl-accepting chemotaxis proteins (MCPs) which act as chemoreceptors in E. coli (Parkinson \& Kofoid, 1992). The similarity in structure and function of MCPs to sensor kinases allows some parallels to be drawn. The MCPs have been found to exist as stable homodimers that signal via an intradimer conformational change (Chervitz \& Falke, 1996; Falke et al., 1997). Similarly, work on EnvZ suggests that interaction between the two membrane-spanning regions is crucial for transmembrane signalling (Tokishita \& Mizuno, 1994). Alternatively, the glutamate residues may be involved in the transport of a charged molecule across the cell membrane, as previously suggested (Lyristis et al., 1994).

Although there are still many details about the VirS/ VirR system that are yet to be determined, the results obtained in this study provide important information about the sensor component. In particular, the significance of the conserved motifs and glutamate residues in the putative membrane-spanning regions has now been established experimentally. Further insights into the structural and functional significance of the $\mathrm{N}$-terminal input domain of VirS will require the experimental verification or modification of the transmembrane model.

\section{ACKNOWLEDGEMENTS}

This research was supported by grants from the Australian National Health and Medical Research Council. J. C. was the recipient of an Australian Postgraduate Award. We thank Dr M. Lyristis for the construction of pJIR1216.

\section{REFERENCES}

Albright, L. M., Huala, E. \& Ausubel, F. M. (1989). Prokaryotic signal transduction mediated by sensor and regulator protein pairs. Annu Rev Genet 23, 311-336. 
Awad, M. M., Bryant, A. E., Stevens, D. L. \& Rood, J. I. (1995). Virulence studies on chromosomal $\alpha$-toxin and $\theta$-toxin mutants constructed by allelic exchange provide genetic evidence for the essential role of $\alpha$-toxin in Clostridium perfringens-mediated gas gangrene. Mol Microbiol 15, 191-202.

Ba-Thein, W., Lyristis, M., Ohtani, K., Nisbet, I. T., Hayashi, H., Rood, J. I. \& Shimizu, T. (1996). The virR/virS locus regulates the transcription of genes encoding extracellular toxin production in Clostridium perfringens. J Bacteriol 178, 2514-2520.

Bannam, T. L. \& Rood, J. I. (1993). Clostridium perfringensEscherichia coli shuttle vectors that carry single antibiotic resistance determinants. Plasmid 29, 223-235.

Bilwes, A. M., Alex, L. A., Crane, B. R. \& Simon, M. I. (1999). Structure of CheA, a signal-transducing histidine kinase. Cell 96, 131-141.

Chervitz, S. A. \& Falke, J. J. (1996). Molecular mechanism of transmembrane signaling by the aspartate receptor: a model. Proc Natl Acad Sci USA 93, 2545-2550.

Claros, M. G. \& von Heijne, G. (1994). TopPred II: an improved software for membrane protein structure predictions. Comput Appl Biosci 10, 685-686.

Deng, W. P. \& Nickoloff, J. A. (1992). Site-directed mutagenesis of virtually any plasmid by eliminating a unique site. Anal Biochem 200, 81-88.

Falke, J. J., Bass, R. B., Butler, S. L., Chervitz, S. A. \& Danielson, M. A. (1997). The two-component signaling pathway of bacterial chemotaxis: a molecular view of signal transduction by receptors, kinases, and adaptation enzymes. Annu Rev Cell Dev Biol 13, 457-512.

Hanahan, D. (1985). Techniques for transformation of E. coli. In DNA Cloning: a Practical Approach, pp. 109-135. Edited by D. M. Glover. Oxford: IRL Press.

Hess, J. F., Bourret, R. B. \& Simon, M. I. (1988). Histidine phosphorylation and phosphoryl group transfer in bacterial chemotaxis. Nature 336, 139-143.

Hsing, W., Russo, F. D., Bernd, K. K. \& Silhavy, T. J. (1998). Mutations that alter the kinase and phosphatase activities of the two-component sensor EnvZ. J Bacteriol 180, 4538-4546.

Island, M. D. \& Kadner, R. J. (1993). Interplay between the membrane-associated $\mathrm{UhpB}$ and $\mathrm{UhpC}$ regulatory proteins. $J$ Bacteriol 175, 5028-5034.

Island, M. D., Wei, B.-Y. \& Kadner, R. J. (1992). Structure and function of the $u h p$ genes for the sugar phosphate transport system in Escherichia coli and Salmonella typhimurium. J Bacteriol 174, 2754-2762.

Jin, S., Roitsch, T., Ankenbauer, R. G., Gordon, M. P. \& Nester, E. W. (1990). The VirA protein of Agrobacterium tumefaciens is autophosphorylated and is essential for vir gene regulation. $J$ Bacteriol 172, 525-530.

Kanamaru, K., Aiba, H. \& Mizuno, T. (1990). Transmembrane signal transduction and osmoregulation in Escherichia coli. I. Analysis by site-directed mutagenesis of the amino acid residues involved in phosphotransfer between the two regulatory components, EnvZ and OmpR. J Biochem 108, 483-487.

La Fontaine, S. \& Rood, J. I. (1996). Organization of ribosomal RNA genes from the footrot pathogen Dichelobacter nodosus. Microbiology 142, 889-899.

Lee, J.-I., Hwang, P. P., Hansen, C. \& Wilson, T. H. (1992). Possible salt bridges between transmembrane $\alpha$-helices of the lactose carrier of Escherichia coli. J Biol Chem 267, 20758-20764.

Lina, G., Jarraud, S., Ji, G., Greenland, T., Pedraza, A., Etienne, J., Novick, R. P. \& Vendenesch, F. (1998). Transmembrane topology and histidine protein kinase activity of $\mathrm{AgrC}$, the $a g r$ signal receptor in Staphylococcus aureus. Mol Microbiol 28, 655-662.

Lyristis, M. (1996). Identification and molecular analysis of the virR/virS regulatory locus from Clostridium perfringens. $\mathrm{PhD}$ thesis, Monash University.

Lyristis, M., Bryant, A. E., Sloan, J., Awad, M. M., Nisbet, I. T., Stevens, D. L. \& Rood, J. I. (1994). Identification and molecular analysis of a locus that regulates extracellular toxin production in Clostridium perfringens. Mol Microbiol 12, 761-777.

Morelle, G. (1989). A plasmid extraction procedure on a miniprep scale. Focus 11, 7-8.

Parkinson, J. S. \& Kofoid, E. C. (1992). Communication modules in bacterial signaling proteins. Annu Rev Genet 26, 71-112.

Rood, J. I. (1983). Transferable tetracycline resistance in Clostridium perfringens strains of porcine origin. Can J Microbiol 29, 1241-1246.

Rood, J. I. \& Cole, S. T. (1991). Molecular genetics and pathogenesis of Clostridium perfringens. Microbiol Rev 55, 621-648.

Rood, J. I. \& Lyristis, M. (1995). Regulation of extracellular toxin production in Clostridium perfringens. Trends Microbiol 3, 192-195.

Rood, J. I., Maher, E. A., Somer, E. B., Campos, E. \& Duncan, C. L. (1978). Isolation and characterization of multiple antibioticresistant Clostridium perfringens strains from porcine feces. Antimicrob Agents Chemother 13, 871-880.

Sambrook, J., Fritsch, E. F. \& Maniatis, T. (1989). Molecular Cloning: a Laboratory Manual, 2nd edn. Cold Spring Harbor, NY : Cold Spring Harbor Laboratory.

Scott, P. T. \& Rood, J. I. (1989). Electroporation-mediated transformation of lysostaphin-treated Clostridium perfringens. Gene 82, 327-333.

Shimizu, T., Ba-Thein, W., Tamaki, M. \& Hayashi, H. (1994). The virR gene, a member of a class of two-component response regulators, regulates the production of perfringolysin $\mathrm{O}$, collagenase, and hemagglutinin in Clostridium perfringens. J Bacteriol 176, 1616-1623.

Smith, M., Jessee, J., Landers, T. \& Jordan, J. (1990). High efficiency bacterial electroporation: $1 \times 10^{10}$ E. coli transformants/mg. Focus 12, 38-40.

Stevens, D. L., Mitten, J. \& Henry, C. (1987). Effects of $\alpha$ and $\theta$ toxins from Clostridium perfringens on human polymorphonuclear leukocytes. J Infect Dis 156, 324-333.

Stock, J. B., Ninfa, A. J. \& Stock, A. M. (1989). Protein phosphorylation and regulation of adaptive responses in bacteria. Microbiol Rev 53, 450-490.

Stock, J. B., Surette, M. G., Levit, M. \& Park, P. (1995). Twocomponent signal transduction systems: structure-function relationships and mechanisms of catalysis. In Two-Component Signal Transduction, pp. 25-51. Edited by J. A. Hoch \& T. J. Silhavy. Washington, DC: American Society for Microbiology.

Tanaka, T., Saha, S. K., Tomomori, C. \& 12 other authors (1998). NMR structure of the histidine kinase domain of the E. coli osmosensor EnvZ. Nature 396, 88-92.

Tokishita, S. \& Mizuno, T. (1994). Transmembrane signal transduction by the Escherichia coli osmotic sensor, EnvZ: intermolecular complementation of transmembrane signaling. Mol Microbiol 13, 435-444.

Uhl, M. A. \& Miller, J. F. (1994). Autophosphorylation and phosphotransfer in the Bordetella pertussis BvgAS signal transduction cascade. Proc Natl Acad Sci USA 91, 1163-1167.

Weinrauch, Y., Penchev, R., Dubnau, E., Smith, I. \& Dubnau, D. 
(1990). A Bacillus subtilis regulatory gene product for genetic competence and sporulation resembles sensor protein members of the bacterial two-component signal-transduction systems. Genes Dev 4, 860-872.

Yang, Y. \& Inouye, M. (1991). Intermolecular complementation between two defective mutant signal-transducing receptors of Escherichia coli. Proc Natl Acad Sci USA 88, 11057-11061.
Yang, Y. \& Inouye, M. (1993). Requirement of both kinase and phosphatase activities of an Escherichia coli receptor (Taz1) for ligand-dependent signal transduction. J Mol Biol 231, 335-342.

Received 14 June 1999; revised 15 September 1999; accepted 25 October 1999. 